\title{
Investigation and Performance Tests of a Designed and Constructed Cylindrical Ionization Chamber (0.6cc) in Iran
}

Mahdi Seifi Moradi* and Mostafa Ghafoori²

${ }^{1}$ Department of Medical Radiation Engineering, Science and Research Branch, Islamic Azad University, Tehran, Iran

${ }^{2}$ Standards Dosimetry Lab, Agricultural, Medical and Industrial Research School, Nuclear Science and Technology Research Institute, Karaj, Iran

\begin{abstract}
There are a number of codes, reports and protocols by national and international organizations, including IAEA, which provide physicists with a systematic approach to dosimetry of photon beams. Most of these dosimetry recommendations have explicitly recognized the advantages of using cylindrical ionization chambers for dosimetry of therapeutic beams, especially for photon beams with energies from kilo voltage (kV) to megavoltage (MV) $\mathrm{x}$-ray. A commercial cylindrical ionization chamber (W-30001) was used as the reference chamber for compare measurements. The homemade $0.6 \mathrm{cc}$ ionization chamber (CC1) have been designed, fabricated, tested and calibrated. Measurements were made using a Farmer type 2670 electrometer together with these chambers. Leakage current, short-term stability, cable effect, repeatability and angular dependence of the CC1 and W-30001 were all tested and found to be in consistence with the international standard IEC 60731. Ion collection efficiency and polarity effect were determined during calibration of the chambers in Co-60. According to the preliminary test results the CC1 homemade chamber is found to be in consistence with the international standard IEC 60731. An advantage of CC1 chamber is a very low leakage current i.e. its specific insulation design and material.
\end{abstract}

Keywords: Ionization chamber; Dosimetry; Calibration; Absorbed dose; Kerma

\section{Introduction}

There are a number of codes, reports and protocols by national and international organizations, including IAEA, which provide physicists with a systematic approach to dosimetry of photon beams $[1,2]$. Most of these dosimetry recommendations have explicitly recognized the advantages of using cylindrical ionization chambers for dosimetry of therapeutic beams, especially for photon beams with energies from kilo voltage $(\mathrm{kV})$ to megavoltage $(\mathrm{MV}) \mathrm{x}$-ray [3]. Radiotherapy machines are widely used in developed countries and to a lesser extent in developing countries. Regarding the possibility of installing more radiotherapy machines in radiotherapy departments in Iran, attempts have been made to construct and make use of a $0.6 \mathrm{cc}$ cylindrical chamber for photon beam dosimetry according to the IAEA TRS No.277 [1], based on air kerma standards and the other IAEA code of practice IAEA TRS No.398 [2], based on absorbed dose to water standards $\left(N_{D, W}\right)$.

\section{Materials and Methods}

\section{Design and fabrication of chamber}

The constructional details of our $0.6 \mathrm{cc}$ cylindrical ion chamber, the CC1, are based on the description given in IAEA TRS 277(based on International Standard IEC 60731:1997, A1:2002 [4]. The design characteristics, mainly the shape and height of the collecting volume, make cylindrical chamber theoretically ideal for ionization measurements in regions with sharp dose gradients in the beam direction or whenever the uncertainty in the position of the effective point of measurement of the ionization chamber is to be minimized. Figure 1 shows different pieces and connectors of the CC1. Figure $1 \mathrm{~A}$ and $\mathrm{B}$ show TNC connector for $0.6 \mathrm{cc}$ ion chamber connection to electrometer, Figure $1 \mathrm{C}$ and $\mathrm{D}$ show $\mathrm{Al}$ and PMMA pieces and the connectors of the chamber, Figure $1 \mathrm{E}$ shows the completed configuration of the fabricated chamber and Figure $1 \mathrm{~F}$ shows the completed configuration of the fabricated chamber plus build up cap for Co-60 measurement in air.
The diagram and basic design characteristics of the CC1 are given in Figure 2 and Table 1. For comparison, Table 1 gives the CC1 dimensions and also those for a commercial cylindrical ionization chamber, the PTW W-30001. Both chambers are not waterproof.

\section{Results}

\section{Preliminary tests}

Leakage current, short-term stability, cable effect, repeatability and angular dependence of the CC1 and W-30001 were all tested and found to be in consistence with the international standard IEC 60731:1997, A1:2002 (ion collection efficiency and polarity effect were determined during calibration of the chambers in Co-60). An electrometer type Farmer 2670 was used for all measurements and tests.

A Picker V9 Co-60 therapy unit was used as a radiation source. For all tests, except for the test of angular dependence and short term stability, the chambers were placed at a depth of $5 \mathrm{~cm}$ in a water phantom. The solid-water phantom dimensions were $20 \mathrm{~cm} \times 20 \mathrm{~cm} \times$ $10 \mathrm{~cm}$ with $5 \mathrm{~cm}$ fixed depth (Figure 3).

\section{Leakage test}

The pre and post-irradiation leakage currents for the CC1 were about $\pm 0.6 \times 10^{-15} \mathrm{~A}$ and $\pm 2 \times 10^{-15} \mathrm{~A}$ respectively. The pre and postleakage currents for W-30001were found to be about $\pm 1 \times 10^{-15} \mathrm{~A}$

\footnotetext{
*Corresponding author: Mahdi Seifi Moradi, Department of Medical Radiation Engineering, Science and Research Branch, Islamic Azad University, Tehran, Iran, E-mail: mahdi_seifi2010@yahoo.com

Received January 30, 2012; Accepted February 20, 2012; Published February 24, 2012

Citation: Moradi MS, Ghafoori M (2012) Investigation and Performance Tests of a Designed and Constructed Cylindrical Ionization Chamber (0.6cc) in Iran. J Nucl Med Radiat Ther S3:003. doi:10.4172/2155-9619.S3-003

Copyright: (c) 2012 Moradi MS, et al. This is an open-access article distributed under the terms of the Creative Commons Attribution License, which permits unrestricted use, distribution, and reproduction in any medium, provided the original author and source are credited.
} 
Citation: Moradi MS, Ghafoori M (2012) Investigation and Performance Tests of a Designed and Constructed Cylindrical lonization Chamber (0.6cc) in Iran. J Nucl Med Radiat Ther S3:003. doi:10.4172/2155-9619.S3-003

Page 2 of 5

and $\pm 5 \times 10^{-15}$ A respectively. Also, within 5 seconds after a 10 -min irradiation, the transient leakage currents of the chamber decreased to less than $1 \%$ of the ionization currents during the irradiation.

\section{Repeatability}

The relative standard deviation calculated from ten successive measurements in different intervals was less than $0.5 \%$ in most cases [6].

\section{Short-term stability}

A reference Sr-90 check source was used for evaluation of short term stability of the chamber response (Figure 4). The difference between check source measurements during 5 month relative to the CC1 response of a reference date was less than $1 \%$ for different intervals.

\section{Cable effect}

The CC1 and W-30001 were irradiated in a rectangular field, $6 \mathrm{~cm}$ $\times 24.5 \mathrm{~cm}$ (at phantom surface) in two configurations. First, the cable was positioned parallel to the larger side of the irradiation field. In the second irradiation, the cable was positioned perpendicular to the larger side of the field. For both configurations, the difference of the collected signal for both chambers was less than $1 \%$.

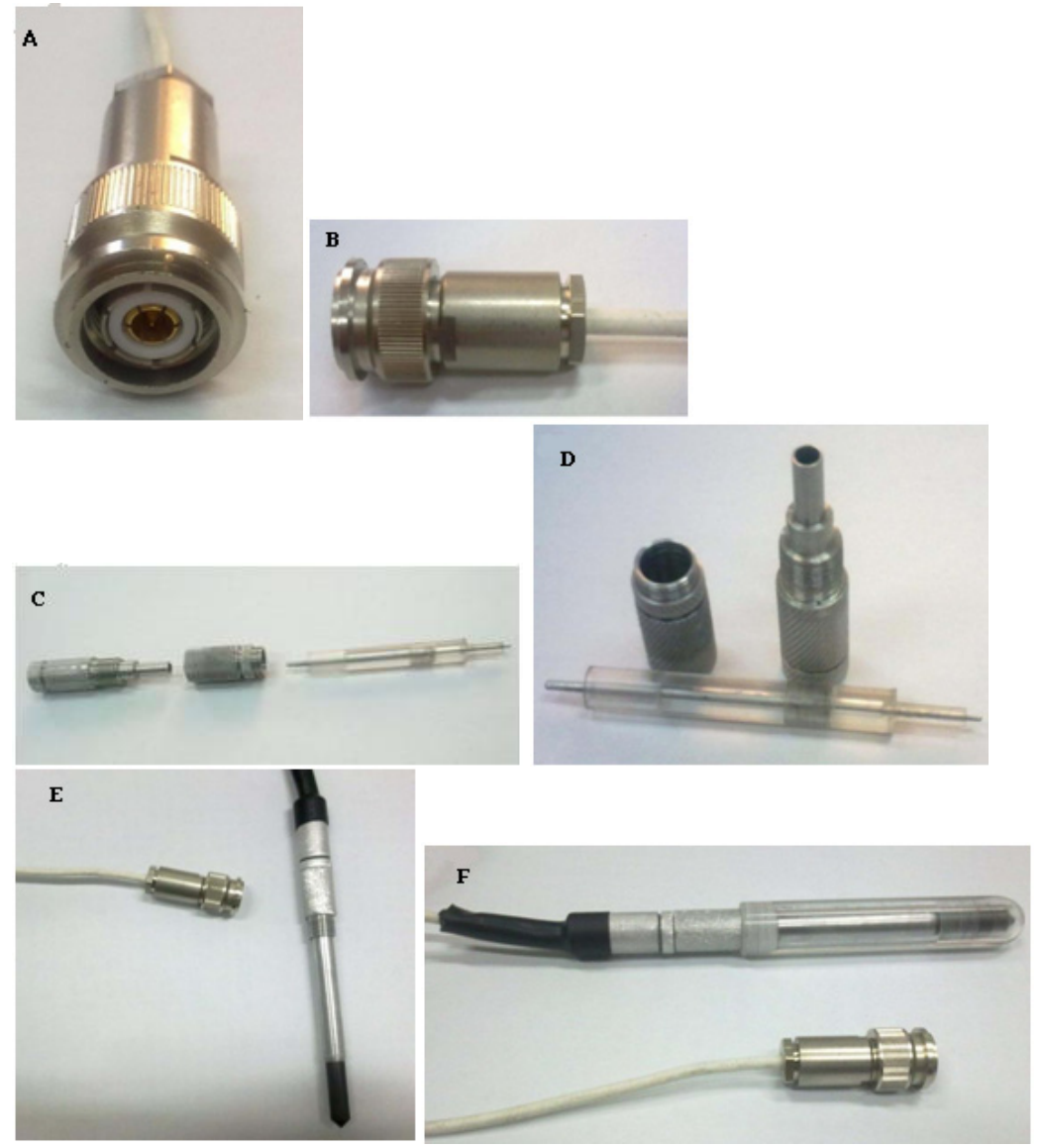

Figure 1: Different pieces and connectors of homemade cylindrical chamber (Figure $1 \mathrm{~A}$ and $\mathrm{B}$ show TNC connector for $0.6 \mathrm{cc}$ ion chamber connection to electrometer, Figure $1 \mathrm{C}$ and $\mathrm{D}$ show $\mathrm{Al}$ and PMMA pieces and the connectors of the chamber, Figure 1E shows the completed configuration of the fabricated chamber and Figure $1 \mathrm{~F}$ shows the completed configuration of the fabricated chamber plus build up cap for Co-60 measurement in air.).

\begin{tabular}{|c|c|c|}
\hline Chamber & CC1 & W-30001 \\
\hline Measuring volume & $0.6 \mathrm{cc}$ & $0.6 \mathrm{cc}$ \\
\hline Wall material and thicknesses & $0.425 \mathrm{~mm}$ graphite & $0.275 \mathrm{~mm}$ PMMA + $0.15 \mathrm{~mm}$ graphite \\
\hline Electrode material & $\mathrm{Al}$ & $\mathrm{Al}$ \\
\hline Build-up cap & Length $89.0 \mathrm{~mm}$, diameter $16.3 \mathrm{~mm}$, (PMMA) & Length $93.0 \mathrm{~mm}$, diameter $16.4 \mathrm{~mm}$, (PMMA) \\
\hline Temperature & $10^{\circ} \mathrm{C}-40^{\circ} \mathrm{C}$ & $10^{\circ} \mathrm{C}-40^{\circ} \mathrm{C}$ \\
\hline Relative humidity & $10 \%-75 \%$ & $10 \%-80 \%\left(<20 \mathrm{~g} / \mathrm{m}^{3}\right)$ \\
\hline Air pressure & (800-1050) hPa (mbar) & (930-1050) hPa (mbar) \\
\hline Reference point & $13 \mathrm{~mm}$ behind chamber top & $13 \mathrm{~mm}$ behind chamber top \\
\hline Connector & TNC & PTW-M, TNC, BNT and BNC + banana \\
\hline
\end{tabular}

Table 1: Dimensions and characteristics, CC1 and W-3000 [5]. 


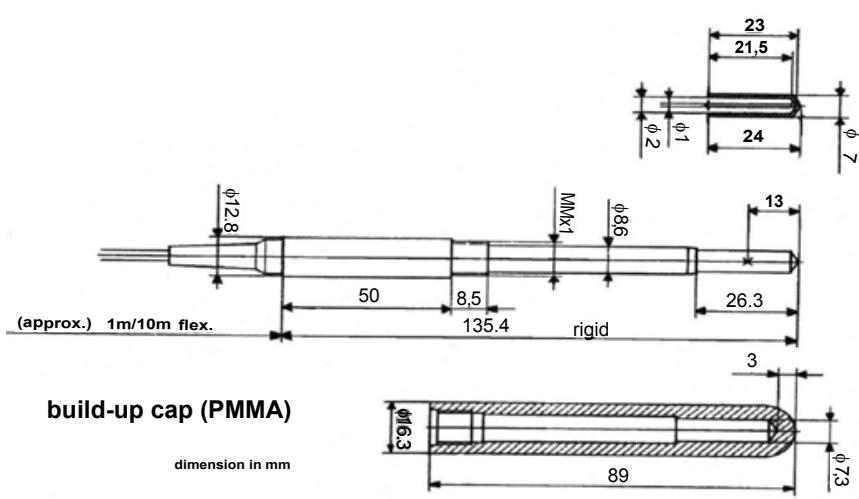

Figure 2: Schematic diagram and dimensions of CC1.

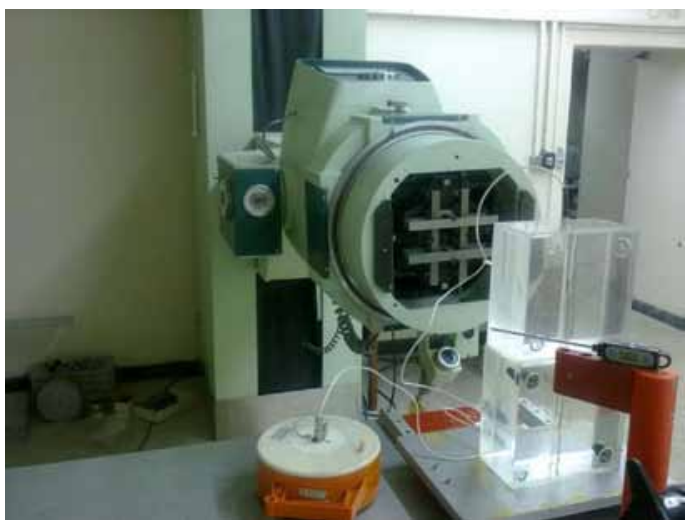

Figure 3: A Co-60 therapy unit, type Picker V9 and the homemade cylindrical chamber in a solid-water phantom.

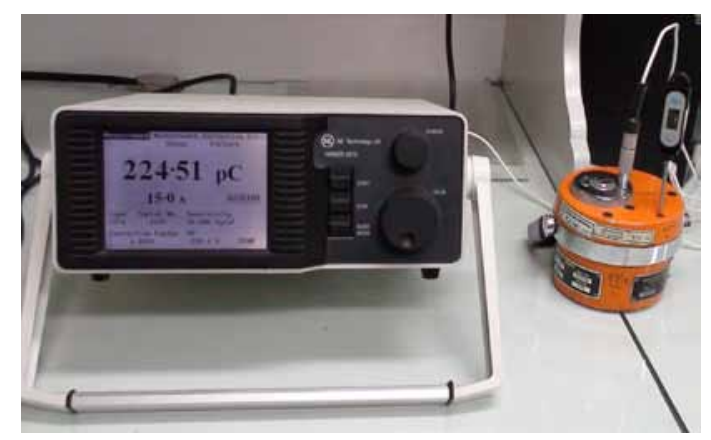

Figure 4: A short-term stability Measurement system (electrometer type Farmer 2670, Sr-90 check source device and our homemade cylindrical chamber).

\section{Angular dependence}

The CC1 and W-30001 were irradiated in air at a distance of $80 \mathrm{~cm}$ from the source. The responses of the chambers were then obtained for several incident angles $(\theta)$ from $-90^{\circ}$ to $+90^{\circ}$. The results of the measurements showed that the response variations of the chambers with respect to the incident angle were not significant. This particular $\mathrm{W}$-30001 chamber displayed a maximum response variation with angle of $0.9 \%$, and this particular CC1 chamber displayed a maximum response variation with angle of $0.3 \%$ (Figure 5 ).

\section{Ion collection efficiency and polarity effect}

Ion recombination corrections were performed during calibration of the CC1 and W-30001, according to the two-voltage method [2]. Two different voltages, $V_{1}=+400 \mathrm{~V}$ and $V_{2}=+100 \mathrm{~V}$, were used to determine the recombination correction factors, $k_{s}$ and two polarizing voltages, $V_{1}=+400 \mathrm{~V}$ and $V_{2}=-400 \mathrm{~V}$ were used in determine the polarity effect $\left(k_{p o l}\right)$. The results are shown in Table 2 and 3 . Relative Response of $\mathrm{CC} 1$ with applied voltage is shown in Figure 6.

$$
\text { for Co-60 } k_{s}=\frac{\left(V_{1} / V_{2}\right)^{2}-1}{\left(V_{1} / V_{2}\right)^{2}-\left(M_{1} / M_{2}\right)}
$$

for megavoltage photon energies $k_{s}=a_{0}+a_{1}\left(M_{1} / M_{2}\right)+$ $a_{2}\left(M_{1} / M_{2}\right)^{2}$ where $M_{1}$ and $M_{2}$ are dosimeter readings at $V_{1}$ and $V_{2}$ respectively and $a_{0}=1.022, a_{1}=-0.363$ and $a_{2}=0.341$ are selected from IAEA TRS-381, Table VII for $V_{1} / V_{2}=4$

\section{Calibration}

There are two general approaches, i.e. air-kerma based and absorbed dose to water based dosimetry codes of practice, to the absorbed dose determination photon beams. The use of an ionization chamber for the determination of the absorbed dose to water in photon beams, requires

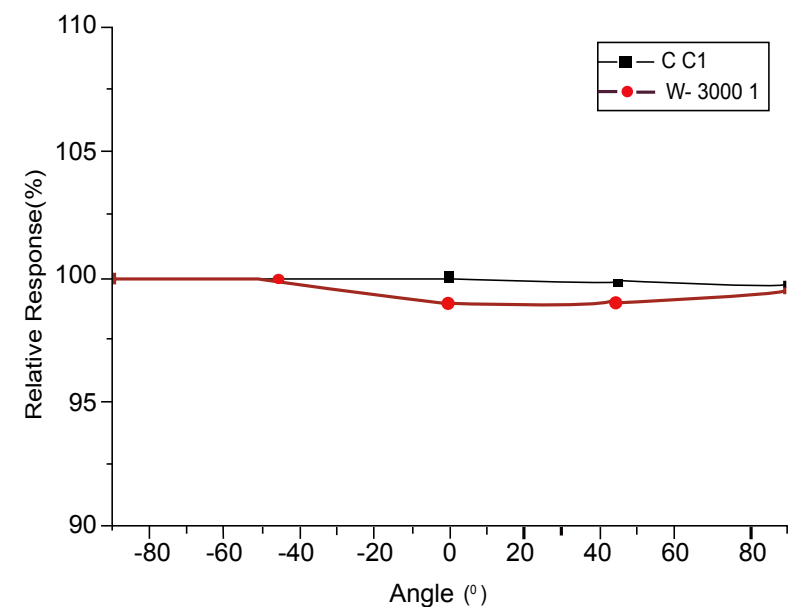

Figure 5: Angular dependence of responses of homemade $0.6 \mathrm{cc}$ cylindrical chamber (CC1) and PTW W-30001 in Co-60 radiation beam.

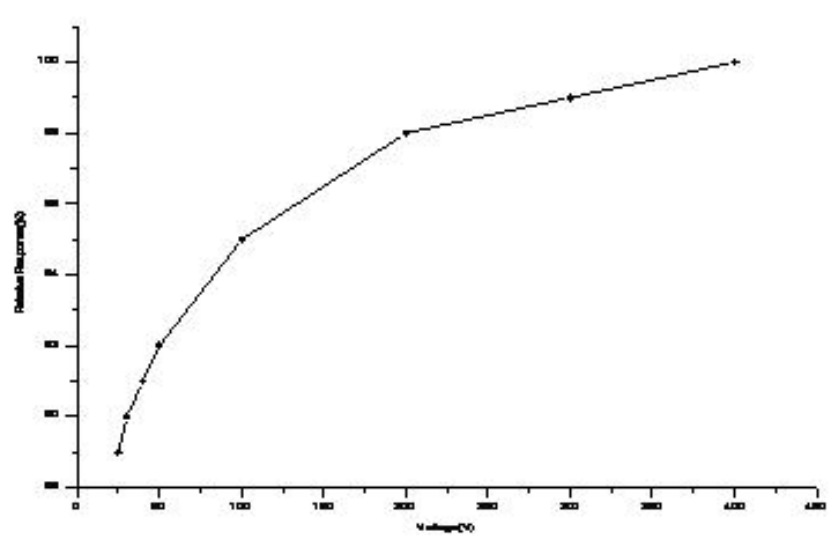

Figure 6: Relative Response of CC1 with applied voltage. 
Citation: Moradi MS, Ghafoori M (2012) Investigation and Performance Tests of a Designed and Constructed Cylindrical lonization Chamber (0.6cc) in Iran. J Nucl Med Radiat Ther S3:003. doi:10.4172/2155-9619.S3-003

\begin{tabular}{|l|l|l|}
\hline & \multicolumn{2}{l|}{$k_{s}$} \\
\hline W-30001 & CC1 \\
\hline $6 \mathrm{MeV}$ & 1.0002 & 1.015 \\
\hline $15 \mathrm{MeV}$ & 1.0008 & 1.031 \\
\hline
\end{tabular}

Table 2: Ion recombination corrections for CC1 and W-30001 chamber.

\begin{tabular}{|l|l|l|}
\hline & $k_{\text {pol }}=\left(M_{-}+M_{+}\right) / 2 M_{-}$ & \\
\cline { 2 - 2 }-30001 & CC1 \\
\hline Co-60 & 0.99 & 0.99 \\
\hline $6 \mathrm{MeV}$ & 0.99 & 0.98 \\
\hline $15 \mathrm{MeV}$ & 0.99 & 0.98 \\
\hline
\end{tabular}

Table 3: polarity effect corrections for CC1 and W-30001 chamber.

the chamber to have either an air kerma calibration factor, $N_{k}$, or an absorbed dose to water calibration factor at the radiation quality $Q$ $N_{D, W, Q}[2]$.

When the chamber has an $N_{k}$ factor, the air kerma ( $\left.K_{\text {air }}\right)$ at the reference depth, $z_{\text {ref }}$, is given by

$$
K_{\text {air }, \text { ref }}=M_{Q} N_{k}
$$

Where $M_{Q}$ is the dosimeter reading corrected for influence quantities.

When the chamber has a calibration factor in terms of absorbed dose to water at the reference quality $Q_{0}, N_{D, W, Q_{0}}$, the absorbed dose to water at the reference depth is given by [7]:

$$
D_{W, Q}\left(z_{r e f}\right)=M_{Q} N_{D, W, Q_{0}} K_{Q, Q_{0}}
$$

Where $K_{Q, Q_{0}}$ is a chamber-specific factor which corrects for difference between the reference beam quality $Q_{0}$ and actual beam quality $Q$.

In order to determine the $N_{k}$ factor of CC1, the $N_{k}$ factor of the reference cylindrical chamber should be known. Therefore we have calculated them by

$$
N_{k}=\frac{K_{\text {air }, \text { ref }}}{M_{Q}}
$$

where $K_{\text {air,ref }}$ is the air kerma measured by the reference chamber. For the Ion chamber PTW W-30001 (\#2108), which is calibrated at the IAEA dosimetry laboratory and $\mathrm{CC} 1$ chamber calibrated against $\mathrm{W}$-30001 the determined air kerma calibration factors $\left(\mathrm{N}_{\mathrm{k}}\right)$ are $49 \pm 1 \%$ $\mathrm{mGy} / \mathrm{nC}$ and $38.02 \pm 1.1 \% \mathrm{mGy} / \mathrm{nC}$ respectively.

In order to calibrate the $\mathrm{CC} 1$, in terms of absorbed dose to water at Co-60, we first used the W-30001 (\#2801) cylindrical chamber, for which the $N_{D, W, C o}$ factor was known. The factors of the CC1 were then determined by comparing their response with that of $\mathrm{W}-30001$ in a water phantom.

\section{The factor of the CC1 was obtained from}

$$
N_{D, W, C o}^{C C 1}=N_{D, W, C o}^{r e f}\left(\frac{M^{r e f}}{M^{C C 1}}\right)
$$

Our reference cylindrical chamber, W-30001 (\#2108), has an absorbed dose to water calibration factor $N_{D, W, C o}=53.6 \mathrm{mGy} / \mathrm{nC} \pm 1.4 \%$ (IAEA, 2003). Using the same value for the ratio $M^{\text {ref }} / \mathrm{M}^{\mathrm{CC} 1}$, the factor for the $\mathrm{CC} 1$ was determined to be

$$
N_{D, W, C o}^{C C 1}=41.08(\mathrm{mGy} / \mathrm{nC}) \pm 1.5 \%
$$

The calibration factor in terms of absorbed dose to water for the chamber under calibration, at the cross-calibration quality $Q_{\text {cross }}$ is given by

$$
N_{D, W, Q_{\text {cross }}}^{w-30001}=\left(M_{\left(Q_{\text {cross }}\right)}^{(N E 2571)}\right) /\left(M_{\left(Q_{\text {cross }}\right)}^{(w-30001)}\right) \cdot K_{Q_{\text {cross }}}^{N E 2571} \cdot C o
$$

where $M_{Q_{\text {cross }}}^{N E 2571}$ and $M_{Q_{\text {coss }}}^{w-30001}$ are dosimeter readings for the reference chamber (NE2571) and the chamber under calibration (W-30001)

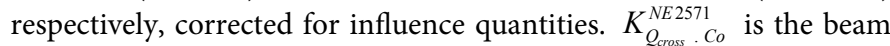
quality correction factor for the reference chamber.

\section{Conclusion}

Cylindrical ionization chambers are found to be very suitable for dosimetry of photon beams in radiotherapy and their use is recommended by most of the dosimetry protocols. Following IAEA dosimetry recommendations in TRS Nos. 277 and 398, we fabricated a $0.6 \mathrm{cc}$ cylindrical chamber and calibrated it in comparison with the responses of calibrated commercial type cylindrical ionization chambers. We also determined and compared absorbed dose in a few photon beam qualities of a medical linac using air-kerma based and absorbed dose to water based methods. This work may be considered as a valuable experience and exercise for SSDL staff who conduct dosimetry and quality audits for radiotherapy centers. We hope our attempts will provide a convenient background for mass production of therapy level ionization chambers. An advantage of CC1 chamber is a very low leakage current i.e. its specific insulation design and material.

\section{Acknowledgment}

The authors of the present paper sincerely and cordially present their special thanks and appreciation to the staff of the SSDL group of Agricultural, Medical and Industrial Research School as well as to the staff of the radiotherapy department of Pars hospital for their kind cooperation and useful guidance.

\section{References}

1. International Atomic Energy Agency (1997) Absorbed dose determination in photon and electron beams: An international code of practice. IAEA TRS No.277 ( $2^{\text {nd }}$ edition) Vienna

2. International Atomic Energy Agency (2000) Absorbed dose determination in external beam radiotherapy: An international code of practice for dosimetry based on standards of absorbed dose to water. IAEA TRS No. 398, Vienna.

3. Khan FM (2003) The physics of radiation therapy: Measurement of ionizing radiation. ( $4^{\text {th }}$ edition) Lippincott Williams \& Wilkins, Philadelphia, USA.

4. British Standards Institute Staff (1997) International Electro technica Commission: Medical electrical equipment dosimeters with ionization chambers as used in radiotherapy. Geneva.

5. PTW Radiation Medicine Quality Assurance (QA) Catalog. Radiation medicine products from PTW-radiation therapy. PTW-Freiburg-Germany, (2008/2009).

6. International Atomic Energy Agency (2009) Calibration of reference dosimeters for external beam radiotherapy. Evaluating the uncertainty. IAEA TRS No.469: $57-61$

7. American Association of Physicists in Medicine TG 21 (1943) A protocol for the determination of absorbed dose from high-energy photon and electron beams. Med Phys 10: 741 